\title{
Morphology Evolution Of ZnO Thin Films Deposited By Nitrogen Mediated Crystallization Method
}

\author{
Iping Suhariadi ${ }^{1, *}$, Masaharu Shiratani ${ }^{2}$, and Naho Itagaki $^{2}$ \\ ${ }^{1}$ Computer Science Department, School of Computer Science, Bina Nusantara University, Jl. K.H. \\ Syahdan No. 09, Palmerah, Jakarta Barat 11480, Indonesia \\ ${ }^{2}$ Graduate School of Information Science and Electrical Engineering, Kyushu University, Fukuoka \\ 819-0395, Japan
}

\begin{abstract}
We study the surface morphology of $\mathrm{ZnO}$ thin films deposited by nitrogen mediated crystallization method utilizing atomic force microscopy as a function of nitrogen flow rates. Initially, the surface morphology of $\mathrm{ZnO}$ thin film deposited without nitrogen exhibits a bumpy surface with spiky grains where the skewness and kurtosis values were found to be 0.48 and 4.80 , respectively. By addition of small amount of nitrogen, the skewness and kurtosis values of the films significantly decrease associated with a flatter topography. Further increase in nitrogen flow rate to $16 \mathrm{sccm}$ has roughened the surface shown mainly by the increase in kurtosis value to be 3.30. These results indicate that the addition of small amount of nitrogen during deposition process has enhanced the adatoms migration on the surface resulting in a superior film with a larger grain size. Two-dimensional power spectral density analysis reveals that all the films have self-affine fractal geometry with total fractal values in the range of 2.14 to above 3.00 .
\end{abstract}

\section{Introduction}

$\mathrm{ZnO}$ thin film has drawn attention due to has many promising features such as a wide direct band gap energy $(\sim 3.3 \mathrm{eV})$ and high room temperature excitonic binding energy (60 meV) [1]. Moreover, the environmentally friendliness and natural abundance of $\mathrm{ZnO}$ make them a strong candidate to replace the use of high cost and poisonous indium doped tin oxide (ITO) [2]. However, $\mathrm{ZnO}$ exhibits disadvantages because its resistivity is higher than that of ITO and strongly depends on film thickness [3]. We have recently overcome the problems by inserting a very thin $\mathrm{ZnO}$ buffer layer deposited by nitrogen mediated crystallization (NMC) between $\mathrm{ZnO}: \mathrm{Al}$ (AZO) and quartz glass substrate [4]. For example, by inserting 10-nmthick NMC-ZnO buffer layer, AZO film with resistivity of $5 \times 10-4 \Omega \mathrm{cm}$ with much lower thickness dependence can be obtained [5].

Nitrogen flow rate control during the deposition has been reported to be one of keys in determining the physical and chemical properties of the films. The introduction small amount

\footnotetext{
*Corresponding author: isuhariadi@binus.edu
} 
of nitrogen during the fabrication of the buffer layer have remarkably improved the surface roughness and increased the grain size of the film and hence leading to the improvement of crytallinity, morphology, and electrical properties of AZO films [6]. Nonetheless, the detail morphological evolution of the $\mathrm{ZnO}$ buffer layers due to the nitrogen addition has not yet been fully understood. Here we study the morphological evolution utilizing power spectral density and scaled height distribution analysis.

\section{Experimental}

$\mathrm{ZnO}$ thin films were deposited on the cleaned quartz glass substrate using RF-magnetron sputtering. The film thickness of each sample was $10 \mathrm{~nm}$. The Ar/N2 flow rate ratios were 24.5/0, 19.5/5, 18.5/6, and 8.5/16 sccm. The detailed experiment was discussed elsewhere [6]. 2D Power Spectral Density Analysis and Height Distribution Analysis were derived from AFM measurement using Veeco Nanoscope II. The scan size was $1 \times 1 \mu \mathrm{m}^{2}$.

\subsection{Height Distribution Analysis}

In the surface analysis, there are two very important parameters that are Root Mean Square (RMS) roughness $(\mathrm{Rq})$ and average roughness $(\mathrm{Ra})$ and. $\mathrm{Ra}$ is the mean height as calculated over the entire measured area which is described as [7]:

$$
\mathrm{R}_{\mathrm{q}}=\sqrt{\frac{1}{\mathrm{~L}} \iint_{0}^{\mathrm{L}} \mathrm{z}^{2}(\mathrm{x}) \mathrm{dx}}
$$

While $\mathrm{Rq}$ is the square root of the distribution of surface height and is tought to be more sensitive than the average roughness for large deviations from the mean line or plane which is defined as:

$$
\mathrm{R}_{\mathrm{a}}=\frac{1}{\mathrm{~L}} \int_{0}^{\mathrm{L}}|\mathrm{z}(\mathrm{x})| \mathrm{dx}
$$

In real practice, the use of $\mathrm{Ra}$ and $\mathrm{Rq}$ is interchangable by ignoring the difference between them. However, this is not necessarily correct for surfaces with more peaks than valley (or vice versa). When the $\mathrm{Rq}$ become more sensitive to the outliers in this case, however, this completely different profiles of the surface cannot be distinguished by Ra.

To sort this problem out, the height distribution is employed which provides a complete specification of random variable, $h(r)$, at position $r$. Even though many surfaces have more or less symmetrical height distribution, nonetheless it has been agreed that the well-known Gaussian distribution is a convenient method to represent such surfaces. The hight asymmetry of the Gaussian distribution was described by statistical parameters such as skewness and kurtosis. As the name signifies, skewness (SK) is a measure of the degree of asymmetry of the distribution, which is normalised as [8]

$$
\mathrm{S}_{\mathrm{K}}=\frac{1}{\mathrm{w}^{3}} \frac{1}{\mathrm{~N}} \sum_{\mathrm{i}=1}^{\mathrm{N}}\left(\mathrm{h}_{\mathrm{i}}-\overline{\mathrm{h}}\right)^{4}
$$

This SK is of importance to characterize the morphological structure of the surfaces that have the same Ra or Rq. The positive and negative SK represent the asymmetric distribution whose tail extending toward more positive or negative height with refer to the mean surface level. This implies that the positive SK indicates that the surface has more bumps while the negative SK indicates that the surface has more holes. Kurtosis $(\mathrm{K})$ describes the entire shape of the 
distribution associated with the level of the randomness of the surface profile relative to that of a Gaussian distribution which is defined as:

$$
\mathrm{K}=\frac{1}{\mathrm{w}^{4}} \frac{1}{\mathrm{~N}} \sum_{\mathrm{i}=1}^{\mathrm{N}}\left(\mathrm{h}_{\mathrm{i}}-\overline{\mathrm{h}}\right)^{4}
$$

For Gaussian associated with perfectly random surface, the distribution the $\mathrm{K}$ is 3 . For $\mathrm{K}<$ 3 , the surface is classified as platykurtic associated with a bumpy surface. For $\mathrm{K}>3$, the surface is classified as leptokurtic associated with a spiky surface.

\subsection{Power Spectral Density}

The scattering theories explain that the amount of optical scatter depends not only on the roughness height of a structure but also on its lateral distribution. Any randomly rough surface can be considered as a Fourier series of sinusoidal waves with different amplitudes, periods, and phases. At a randomly rough surface many different spatial spatial frequency are present. Power spectral density is a technique based on fast Fourier transform algorithm, which is very useful to quantitatively analyze microstructure as a function of spatial frequency. In general, there are two common PSD analyses that are one-dimensional (1D) and two-dimensional (2D) PSD. The 1D PSD is calculated from the scan line profile measurement of AFM result that can be written as [9]

$$
\operatorname{PSD}(\mathrm{k})=\frac{1}{\mathrm{~L}}\left[\int_{0}^{\mathrm{L}} \mathrm{h}(\mathrm{x}) \mathrm{e}^{\mathrm{ikx})} \mathrm{dx}\right]^{2}
$$

Where $\mathrm{k}$ and $\mathrm{L}$ and are the spatial frequency and the total length of the scanned area. By extracting the scaling behavior, the PSD is then can be written as:

$$
\operatorname{PSD}(\mathrm{k}) \propto \mathrm{k}^{-\gamma}
$$

Here $\gamma$ is related to height correlation function exponent through $\alpha=(\gamma-d) / 2$. The unit of 1D-PSD is in (length) ${ }^{3}$. In the case 2D-PSD the mathematical equation can be written as [10]:

$$
\operatorname{PSD}_{2 D}\left(\mathrm{f}_{\mathrm{x}}, \mathrm{f}_{\mathrm{y}}\right)=\frac{1}{\mathrm{~L}^{2}}\left[\sum_{\mathrm{m}=1}^{\mathrm{N}} \sum_{\mathrm{n}=1}^{\mathrm{N}} \mathrm{Z}_{\mathrm{mn}} \mathrm{e}^{-2 \pi \mathrm{i} \Delta \mathrm{L}\left(\mathrm{f}_{\mathrm{x}} \mathrm{m}+\mathrm{f}_{\mathrm{n}} \mathrm{n}\right)}(\Delta \mathrm{L})^{2}\right]^{2}
$$

where $\mathrm{N}$ is the number of data points per line and row, $\mathrm{Z}_{\mathrm{mn}}$ is the profile height at position $(\mathrm{m}, \mathrm{n}), \mathrm{fx}$ and fy correspond to the spatial frequency in the $\mathrm{x}$ - and $\mathrm{y}$ - direction, respectively, and $\Delta \mathrm{L}=\mathrm{N} / \mathrm{L}$ is the distance of sampling. For fractals and superstructures characterization, especially for the thin film coating, the 2D-PSD can be written by considering only the pure film as [11]:

$$
\operatorname{PSD}_{\mathrm{ABC}}=\frac{\mathrm{J}}{\left(1+\mathrm{K}^{2} \mathrm{f}^{2}\right)^{(\gamma+1) / 2}}
$$

With $\mathrm{J}$ defines as the magnitude at low spatial frequency which is related to the amplitude of the rough surface, $\mathrm{K}$ is the "knee" which is defined as the slope of a line connecting two point on the surface, being associated with the mean grain size, and $\gamma$ is the slope at high spatial frequency that gives the nature of the local roughness, respectively. The profile's fractal dimension $\mathrm{D}_{\mathrm{f}}$ can be expressed as

$$
D_{f}=\frac{(7-\gamma)}{2}
$$

The total fractal dimension then given by [12] 


$$
D_{S}=D_{f}+1
$$

\section{Results and Discussion}

Figure 1(a) shows the scaled height distribution of $\mathrm{ZnO}$ buffer layers as a parameter of nitrogen flow rate. The kurtosis and skewness values deduced from scaled height distribution are shown in the Figure 1(b) and 1(c), respectively.
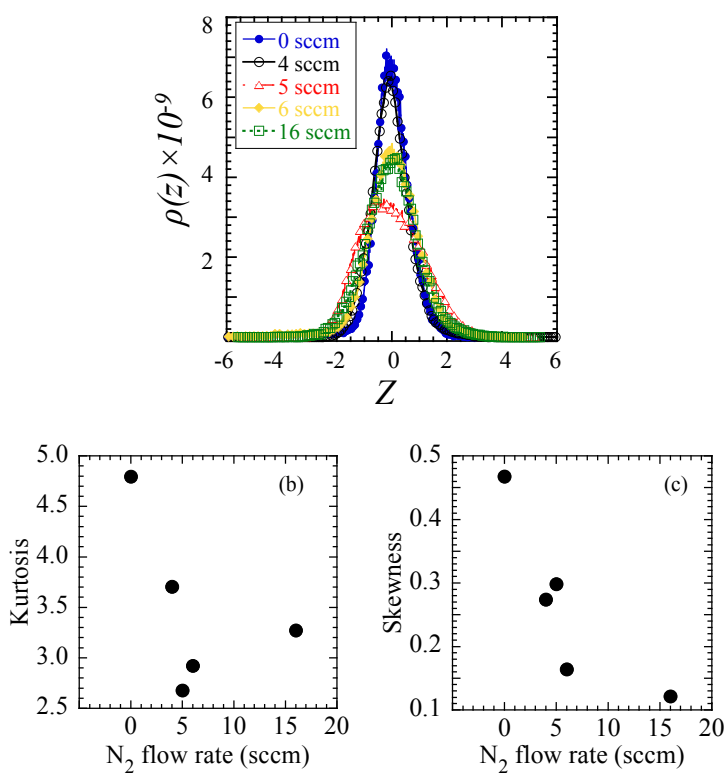

Fig. 1. (a) Scaled height distribution; (b) skewness; and (c) kurtosis of $\mathrm{ZnO}$ deposited as a function of nitrogen flow rate.

Initially, the surface morphology of $\mathrm{ZnO}$ thin film deposited without nitrogen exhibits more bumps with spiky surface where the skewness and kurtosis values were found to be 0.48 and 4.80 , respectively. At this stage, the film is classified as platykurtic. By addition small amount of nitrogen, skewness and kurtosis values of the films decrease to near Gaussian distribution indicating that the surface morphology has transformed from platykurtic to leptokurtic where the bumps decrease and the spiky surfaces become mild surfaces associated with a flatter topography. Nonetheless, further increase in nitrogen flow rate to $16 \mathrm{sccm}$ has roughened the surface shown mainly by the increase in kurtosis value to be 3.30. It is thought that the growth of $\mathrm{ZnO}$ follows ballistic deposition mode where the high energetic ablated species from the target sticks whereupon it lands. As a result, the atoms have a low diffusion rate and nucleate instantaneously leading to a rougher surface morphology. The addition of small amount of nitrogen during deposition has inhibited the nucleation by nitrogen adsorption-desorption mechanism enhancing the adatoms migration on the surface resulting in a superior film with a larger grain size. 


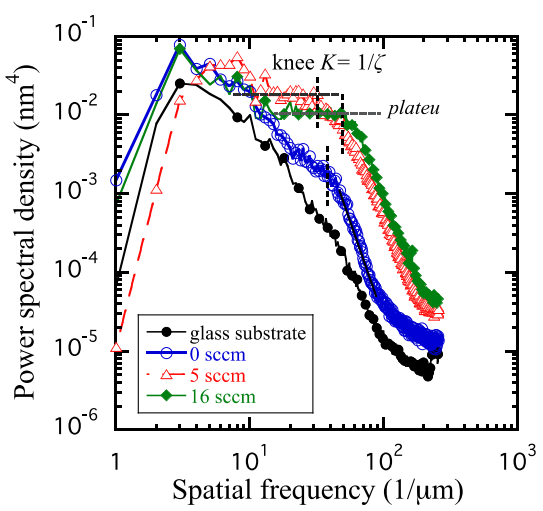

Fig. 2 2D-PSD of $\mathrm{ZnO}$ films deposited at various nitrogen flow rate ratio

Figure 2 shows the two-dimensional (2D) power spectral density of the $\mathrm{ZnO}$ thin films deposited at nitrogen flow rate of 0,5 , and $16 \mathrm{sccm}$. The 2D-PSD plot of glass substrate is also presented as comparison. From Fig. 2 it can be observed that there is no peak in the high spatial frequency region indicating that all films have self-affine surface structure associated with randomly rough surface. This self-affine structures was expected because amorphous substrate was used in our experiments. 2D-PSDs of all films show power law inverse behavior particularly at high spatial frequency region indicating the existence of fractal component in the surface morphology [11]. The values of fractal dimension $\mathrm{D}_{\mathrm{f}}$ and $\mathrm{D}_{\mathrm{S}}$, roughness exponent $\alpha$, and lateral correlation length $\zeta$ deduced from the slope of the spectrum are displayed in Table 1. In the 2D-PSD spectra of Fig. 2, there is no cutoff to zero slope (plateu) for glass substrate indicating that the film topography consists of two surface geometry with $D_{\mathrm{S}}$ values were 2.61 and $>3.00$ associated with near extreme and extreme fractals, respectively. The similar pattern to that of glass substrate is observed for $\mathrm{ZnO}$ film deposited at nitrogen flow rate of $0 \mathrm{sccm}$ with $\mathrm{D}_{\mathrm{s}}$ values of 2.14 and $>3.00$ associated with marginal and extreme fractals.

Table 1. Fractal, roughness exponent, and lateral correlation length parameters of $\mathrm{ZnO}$ deposited at various nitrogen flow rate ratio.

\begin{tabular}{|c|c|c|c|c|c|c|c|c|c|}
\hline \multirow{2}{*}{$\mathrm{N}_{2}$ flow rate $(\mathrm{sccm})$} & \multicolumn{2}{|c|}{$\gamma$} & \multicolumn{2}{|c|}{$\mathrm{D}_{\mathrm{f}}$} & \multicolumn{2}{|c|}{$\mathrm{D}_{\mathrm{S}}$} & \multicolumn{2}{|c|}{$\alpha$} & \multirow[t]{2}{*}{$\zeta(\mathrm{nm})$} \\
\hline & $\gamma_{1}$ & $\gamma_{2}$ & $\mathrm{D}_{\mathrm{f}} 1$ & $\mathrm{D}_{\mathrm{f}} 2$ & $\mathrm{D}_{\mathrm{S}} 1$ & $\mathrm{D}_{\mathrm{S}} 2$ & $\alpha_{1}$ & $\alpha_{2}$ & \\
\hline 0 & 4.72 & 1.61 & 1.14 & 2.70 & 2.14 & $>3.00$ & 1.86 & 0.30 & 26.10 \\
\hline 5 & 4.15 & - & 1.42 & - & 2.42 & - & 1.58 & - & 31.02 \\
\hline 16 & 4.16 & - & 1.42 & - & 2.42 & - & 1.58 & - & 20.33 \\
\hline
\end{tabular}


The roughness exponent values $\alpha=1.86$ and $\alpha=0.3$ suggest that the anisotropic and the NN ballistic growths were the two main competing mechanisms in the film [13]. In contrast, the $\mathrm{ZnO}$ films deposited with nitrogen flow rates of 5 and $16 \mathrm{sccm}$ exhibit only brownian fractal with $D_{s}$ of 2.42 and roughness exponent $\alpha$ of 1.58 regardless of the amount of nitrogen indicating that local and non-local continuum effects have dominantly contributed to the roughness. Due to the sample holder was rotated during the deposition, it is very unlikely that shadowing effects to exist in the film. Hence, the roughening effect in the $\mathrm{ZnO}$ film deposited with nitrogen is inferred mainly due to surface diffussion and grain growth. From the position of knee in Fig. 2 it can be seen that $\mathrm{ZnO}$ film deposited with nitrogen at $5 \mathrm{sccm}$ has a larger lateral correlation length value $\zeta$ associated with a higher surface diffusion and thus a larger mean grain size.

\section{Conclusions}

We have reported the surface characterization of $\mathrm{ZnO}$ thin film deposited at various nitrogen flow rate ratio utilizing scaled height distribution analysis and two-dimensional power spectral density. By this approach, quantitative characterization of the surface morphology can be obtained. We found that the surface morphology of the $\mathrm{ZnO}$ film evolved by the addition of nitrogen during the growth. The high quality surface structure was obtained for the film deposited with small amount of nitrogen flow rate.

\section{References}

1. A. Janotti and C. G. Vand de Walle. Rep. Prog. Phys. 72, 126501 (2009)

2. S. Li, J. Wen, X. Mo, H. Long, H. Wang, J. Wang, G. Fang. J. Power Source 256, 206211 (2014)

3. T. Yamada, A. Miyake, S. Kishimoto, H. Makino, N. Yamamoto, T. Yamamoto, Appl. Phys. Lett. 91, 051915 (2007)

4. D. C. Look, K. D. Leedy, A. Kiefer, B. Claflin, N. Itagaki, K. Matsushima, I. Suhariadi. Opt. Eng. 52(3), 033801 (2013)

5. N. Itagaki, K. Kuwahara, K. Nakahara, D. Yamashita, G. Uchida, K. Koga, M. Shiratani. Appl. Phys. Exp. 4, 011101 (2011)

6. I. Suhariadi, K. Oshikawa, K. Kuwahara, K. Matsushima, D. Yamashita, G. Uchida, K. Koga, M. Shiratani, N. Itagaki. Jpn. J. Appl. Phys. 52, 11 NB03 (2013)

7. S. S. Kim and B. T. Lee. Thin Solid Films 446, 307 (2004)

8. O. Lupan, T. Pauporte, L. Chow, B. Viana, F. Pelle, L. K. Ono, B. R. Cuenya, and H. Heinrich. Appl. Surf. Sci. 256, 1895 (2010)

9. K. Zhu, V. Kuryatkov, B. Borisov, J. Yun, G. Kipshidze, S. A. Nikishin, H. Temkin, D. Aurongzeb, M. Holtz. J. Appl. Phys. 95, 4635 (2004)

10. T. Itoh and N. Yamauchi. Appl. Surf. Sci. 253, 6196 (2007)

11. N. K. Sahoo, S. Thakur, R. B. Tokas. Thin Solid Films 503, 85 (2006)

12. C. J. Buchko, K. M. Kozloff, D. C. Martin. Biomaterials 22, 1289-1300 (2001)

13. Nie, Man. 2014. Growth and morphology evolution of semiconducting oxides and sulfides prepared by magnetron sputtering. Dissertation. Technical University Berlin, Berlin, Germany. 